\title{
IMAGE DEHAZING BASED ON MULTISPECTRAL POLARIZATION IMAGING METHOD IN DIFFERENT DETECTION MODES
}

\author{
Y. Zhang ${ }^{1, *}$, L. Y. Luo ${ }^{1}$, H. J. Zhao ${ }^{1}$, R. D. Qiu ${ }^{1}$, Y. S. Ying ${ }^{1}$ \\ ${ }^{1}$ School of Instrumentation Science \& Opto-electronics Engineering, Beihang University, No. 37 Xueyuan Road, Haidian District, \\ Beijing, 100191, China - (yingzhang, luoly2017, gongxunbuaa, sy1817406, yingyinshuo) @ buaa.edu.cn
}

KEY WORDS: Polarization detection, Image dehazing, Near-earth remote sensing, Polarization spectral analysis, Airlight polarization model, Polarization-based dehazing

\begin{abstract}
:
In haze, the quality of the images is degraded due to the scattering of atmospheric aerosol particles in near-earth remote sensing. Therefore, how to effectively remove the influence of haze and improve image quality has been a hot issue. A polarization spectral image dehazing method is proposed here. A multi-spectral full-polarization imager was used to detect the polarization spectral images of ground objects. Firstly, the maximum- and minimum-intensity polarization images were obtained from the relationship between the Stokes vector and the Mueller matrix. Secondly, the airlight polarization model was utilized to estimate the airlight radiance at an infinite distance and the degree of polarization of the airlight. At last, the atmospheric attenuation model was used to obtain dehazed images. The results proved that our proposed image dehazing method can achieve substantial improvements on the detail recovery, not only in vertical downward detection mode, but also in horizontal detection mode.
\end{abstract}

\section{INTRODUCTION}

The worsening air pollution is causing serious haze problems, the quality of the images obtained by near-earth remote sensing imaging systems is seriously degraded due to the scattering of atmospheric aerosol particles. Hence, there is a hot issue that removing the influence of haze and improving image quality in the field of remote sensing and image processing.

Recent decades, a large number of methods have been proposed for haze removal from images, which can be divided into two categories: image enhanced dehazing method and physical model based dehazing method. The image enhancement methods, such as linear transformation(Tarel and Hautiere, 2009), structure preserving (Qi et al., 2015) and dark channel prior (He et al., 2011), which focus on the haze-degraded image itself without considering the physical process of image degeneration, use computer vision techniques to enhance the image contrast, the loss of some detail information is inevitable. Polarization image dehazing is the method based on physical model (Schechner and Averbuch, 2007; Schechner et al., 2001), which recovers the image based on two images captured at orthogonal polarization states. The polarization image dehazing method, which utilizes the polarization differences between the airlight and the direct light to extract the object radiance from the total intensity, can restore the detailed information of the image (Qu and Zou, 2017). Based on Schechner's method, various improved methods have been proposed to obtain dehazing images. However, the method still suffers from some problems: the estimates of the airlight radiance at an infinite distance $\left(A_{\infty}\right)$ and the degree of polarization of the airlight $(p)$ rely on the sky area or any similar objects in the image. However, when there is no sky area in the image or certain high-brightness objects and strong light sources exist in the image, an inaccurate selection of the sky area will yield errors and affect the dehazed image.
The research on polarization dehazing has mainly been focused on visible-RGB images at present, the image dehazing technique based on polarization spectral image has been reported rarely. The object radiance's ability to penetrate the haze particles has a huge difference under different spectral channels, it is of practical significance to combine the polarimetric dehazing method with spectral analyses (Xia and Liu, 2016). The multi-spectral fullpolarization imager based on Liquid Crystal Variable Retarders (LCVR) used in this paper can obtain spectral information and polarimetric information simultaneously (Zhang et al., 2011). In this paper, we proposed a polarization dehazing method based on atmospheric attenuation model by polarization spectral images. The verified experiments are performed and the results show that this method can effectively improve the polarization image quality in haze. Under different detection modes, the dehazing process can increase image contrast differently under various wavelengths. This method can make it possible for the multispectral full-polarization imager to work under hazy weather.

\section{RELATED WORKS}

\subsection{The Polarization-based Dehazing}

The total intensity of the light received by the imager under hazy weather can be divided into two parts: one part is the direct light reflected from the object; the other part generated is the airlight scattered by haze particles. Under hazy weather, the scattering of aerosol will add unwanted airlight into the total intensity of light and the absorption of aerosol will attenuate the reflection of the scene. The direct light reflected from the scene is non-polarized light which will decrease with the observation distance, meanwhile, the airlight scattered from the haze particles is partial polarized which will increase with the observation distance. Schechner and Nayer (Schechner et al., 2003) proposed a widely used polarization-based dehazing model and the object radiance after the image dehazing can be expressed as

\footnotetext{
* Corresponding author
} 


$$
L^{\text {object }}=\frac{I^{\perp}+I^{\|}-\left(I^{\perp}-I^{\|}\right) / p}{1-\left(I^{\perp}-I^{\|}\right) / p A_{\infty}}
$$

where $\quad L^{\text {object }}=$ object radiance

$I^{\perp}=$ image taken when the polarizer is perpendicular to the plane of the incidence

$I^{\|}=$image taken when the orientation of the polarizer is parallel with the plane of the incidence

$p=$ airlight degree of polarization

$A_{\infty}=$ airlight radiance at an infinite distance.

\subsection{Estimate of $A$}

Schechner (Schechner et al., 2003) chose the sky area with the highest luminance value in the image to estimate $A_{\infty}$. In order to avoid the influence of the deviation of the sky area selection on the dehazed image, Tan (Tan, 2008) and Tarel (Tarel and Hautiere, 2009) proposed subjecting the hazy image to whitebalance pre-treatment with the chroma pre-set to $1 . \mathrm{He}$ (He et al., 2011) proposed a dehazing algorithm based on dark-channel prior, which can eliminate the effect of high-brightness objects and strong light sources during estimating $A_{\infty}$. Zhu (Zhu et al., 2015) speculated that estimating airlight $A_{\infty}$ in the far-field areas of the image can reduce the effect of direct transmission $D$ and obtain $A_{\infty}$ values closer to the actual airlight value.

\subsection{Calculation of $p$}

Schechner (Schechner et al., 2003) rotated a linear polarizer to acquire the maximum- and minimum-intensity images and estimated the $p$ by using the polarization difference information in the sky area. However, if the choice of the sky area is unsuitable or if there is no sky area in the image, this cannot compute the $p$. Namer (Namer et al., 2009) proposed method that $p$ can be obtained even with a non-sky image which should present similar objects in the scene. This method solves the problem of the sky-area dependence. Liang (Liang et al., 2015) solved the Stokes vector of airlight with four different polarization-angle images and then computed the $p$ with the pixel corresponding to the polarization angle of the maximum probability and selected the maximum value in these pixels as $p$.

In order to require the dehazing image of the non-sky area and overcome the problem of high-brightness object interference, we proposed a method of estimating $A_{\infty}$ and $p$ based on the airlight polarization model without the need for any local area in the image.

\section{PROPOSED POLARIZATION-BASED DEHAZING METHOD}

To compare with the traditional polarization-based dehazing, we proposed a new approach to estimate the airlight degree of polarization $(p)$ and the airlight radiance at an infinite distance $\left(A_{\infty}\right)$. The flow chart of our dehazing method is shown in Fig. 1. First, we require four different polarization images of the same band using the multi-spectral full-polarization imager, and we obtain the maximum- and minimum-intensity images according to the relationship of the Stokes parameters and the Mueller matrix. Second, the airlight polarization model based on the RT3 vector radiative transfer model is used to simulate the distribution of the polarization state of the airlight, and then $p$ and $A_{\infty}$ are calculated on these data. Next, a bias factor $\varepsilon$ and a correction factor $\delta$ are introduced to revise $p$ and $A_{\infty}$, respectively. Finally, the maximum- and minimum-intensity images, the airlight degree of polarization and the airlight radiance at an infinite distance are processed by the atmospheric attenuation model to obtain a dehazed image.

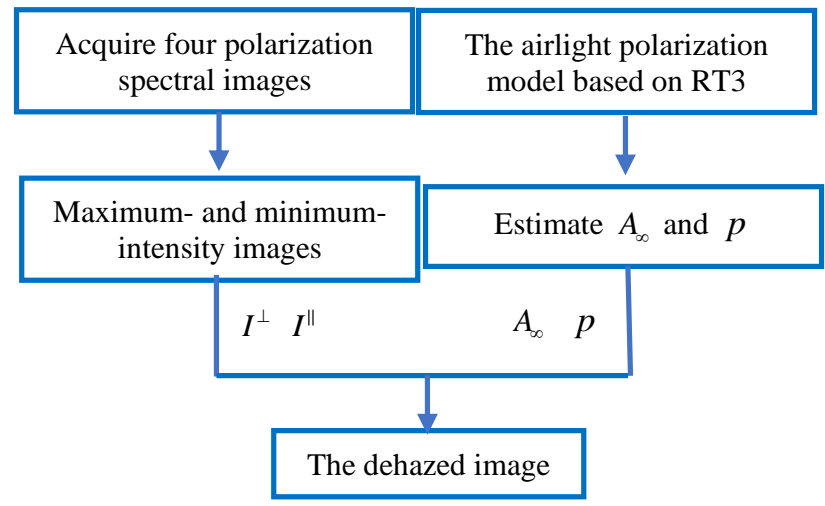

Figure 1. Flow chart of proposed dehazing process.

\subsection{Estimation of Maximum- and Minimum-intensity Images}

The total intensity of the light received by the polarization imager under hazy weather changes with the orientation angle of the polarizer $\alpha$, when the orientation of the polarizer is parallel with the plane of incidence ( $\alpha=\theta^{\|}$), the intensity of the airlight scattered from haze particles $A^{\|}$is the smallest which represents the best state of the original image, when the orientation of the polarizer is perpendicular to the plane of incidence $\left(\alpha=\theta^{\perp}\right)$, the intensity of the airlight scattered from haze particles $A^{\perp}$ is the largest which represents the worst state of the original image.

Based on the Stokes parameters and the Mueller matrix ( $\mathrm{Qu}$ and Zou, 2017), a linear polarizer model with polarization angle of $\alpha$ is established as follows:

$$
\left(\begin{array}{c}
I_{\text {out }} \\
Q_{\text {out }} \\
U_{\text {out }} \\
V_{\text {out }}
\end{array}\right)=M \cdot\left(\begin{array}{c}
I_{\text {in }} \\
Q_{\text {in }} \\
U_{\text {in }} \\
V_{\text {in }}
\end{array}\right)=\frac{1}{2}\left(\begin{array}{c}
I_{\text {in }}+Q_{\text {in }} \cdot \cos 2 \alpha+U_{\text {in }} \cdot \sin 2 \alpha \\
I_{\text {in }} \cdot \cos 2 \alpha+Q_{\text {in }} \cdot \cos ^{2} 2 \alpha+U_{\text {in }} \cdot \cos 2 \alpha \sin 2 \alpha \\
I_{\text {in }} \cdot \sin 2 \alpha+Q_{\text {in }} \cdot \cos 2 \alpha \sin 2 \alpha+U_{\text {in }} \cdot \sin ^{2} 2 \alpha \\
0
\end{array}\right)
$$

where $\left(\begin{array}{llll}I_{\text {out }} & Q_{\text {out }} & U_{\text {out }} & V_{\text {out }}\end{array}\right)^{T}=$ stokes parameters of emergent light

$\left(\begin{array}{llll}I_{\text {in }} & Q_{i n} & U_{\text {in }} & V_{i n}\end{array}\right)^{T}=$ stokes parameters of incidence light

$M=$ Mueller matrix

$\alpha=$ polarization angle

From Eq. (2), the light intensity with polarization angle of $\alpha$ can be expressed as

$$
I_{\text {out }}=I_{\text {in }}+Q_{\text {in }} \cdot \cos 2 \alpha+U_{\text {in }} \cdot \sin 2 \alpha
$$

In our study, we obtain four different polarization images using the full-polarization imager that can be used to solve for the 
polarization state of the incidence light $\left(\begin{array}{llll}I_{i n} & Q_{i n} & U_{i n} & V_{i n}\end{array}\right)^{T}$. However, the circular-polarized component in the polarized light is sufficiently small for it to be neglected ( $V=0)$. After the Stokes vector is known, we can obtain the maximum- and minimum-intensity images for $\alpha=\theta^{\perp}$ and $\alpha=\theta^{\|}\left(\theta^{\|}=\theta^{\perp}+\frac{\pi}{2}\right)$ by means of the derivation method. Settings $\alpha=\theta^{\perp}$ and $\alpha=\theta^{\|}$ correspond to the maximum- and minimum-intensity images $\left(I^{\perp}\right.$ and $\left.I^{\|}\right)$, respectively. The four polarization images obtained by imager, maximum-intensity image and minimum-intensity image are shown in Fig. 2.

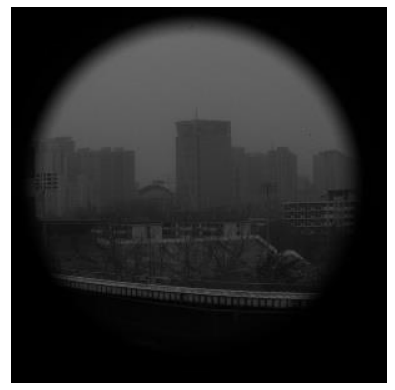

(a)

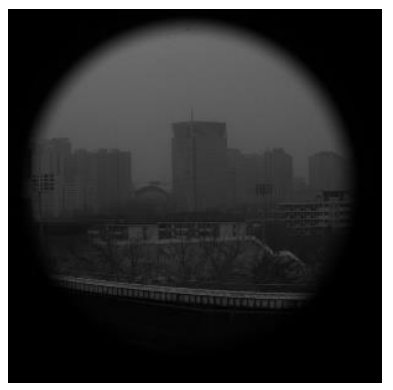

(c)

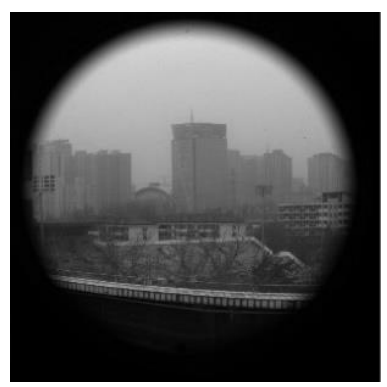

(e)

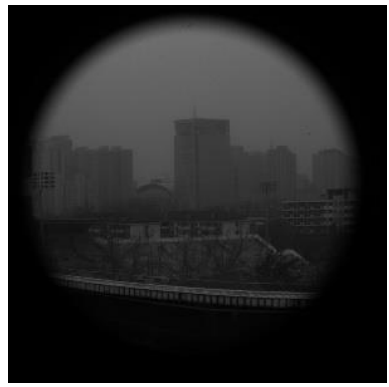

(b)

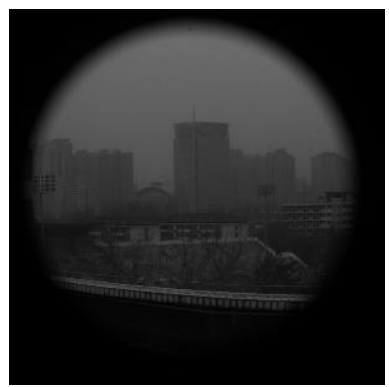

(d)

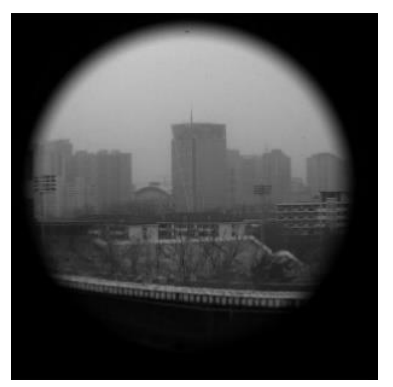

(f)
Figure 2. Estimation of maximum- and minimum-intensity images. (a), (b), (c) and (d) four different original haze polarization images in $530 \mathrm{~nm}$, (e) maximum-intensity image, (f) minimum-intensity image

\subsection{Estimation of $A_{\infty}$ and $p$}

The airlight radiance at infinite distance $A_{\infty}$ and the degree of polarization of the airlight $p$ are the two global constants in a homogeneous atmosphere. In this paper, the $A_{\infty}$ and $p$ are estimated by the airlight polarization model based on RT3 atmospheric radiative transfer model
3.2.1 The Airlight Polarization Model: The airlight polarization models can simulate the polarization state distribution of the airlight, and are key tools to study the quantitative relationship between the polarization state distribution of the airlight and the characteristic parameters of the atmosphere. Zhang (Zhang et al., 2017) proposed a model that can simulate the distribution of the polarization state of the whole sky under various weather conditions. The model is based on the RT3 vector radiative transfer model, which solves the problem of radiation transmission in the atmosphere using adding and doubling approach. For the complex atmospheric stratification information in the actual situation, this method uses a simplified three-layer atmospheric layering, which adds the cloud layer between the basis of two atmospheric layers. And then Rayleigh scattering, Mie scattering theory and T matrix method are used to solve the scattering characteristics of various small molecules, aerosols and cloud particles. The simplified calculation of complex atmospheric stratification information is realized. Lastly combined with a few of ground observation data, the model can calculate the polarization distribution of airlight under various weather conditions. Only the simulation result of the sunny day is shown in the reference, but the polarization distribution of airlight in haze weather has not been verified. We used the multispectral full-polarization imager for all airlight detection experiments and obtained atmospheric aerosol optical thickness measured by a sun photometer and then calculated the solar zenith angle by detecting time. Other data are selected in the reference. The measurements and this model simulation results of airlight DOP is shown in Fig. 3.

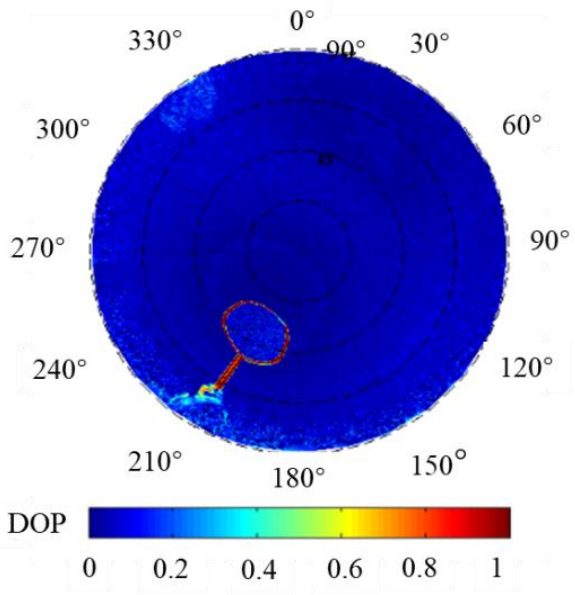

(a)

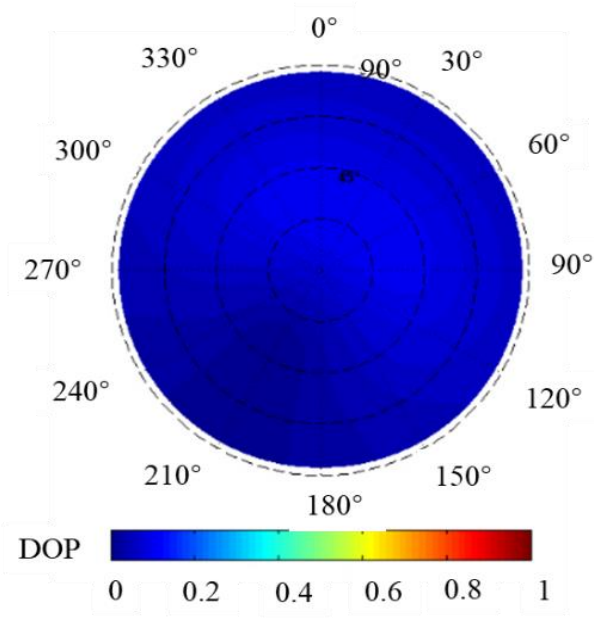

(b) 


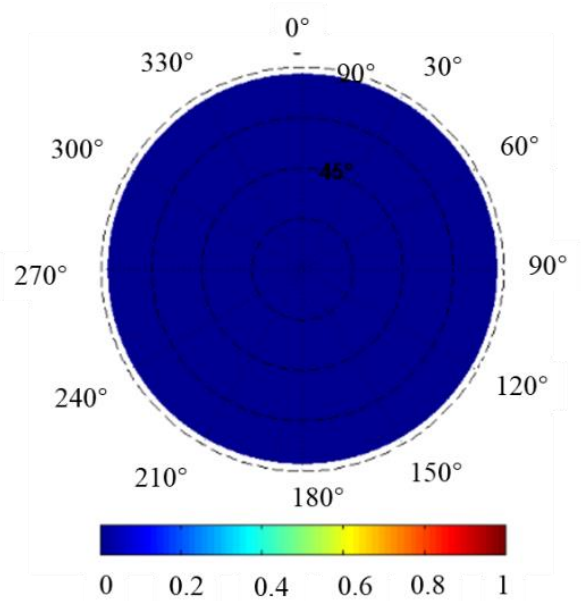

(c)

Figure 3. Measurement and the model simulation result of airlight DOP in 530nm. (a) measurement result, (b) the model simulation result, (c) the relative error of two results

From the Fig. 3, we can observe that the model simulation result is almost identical to the actual situation. So, the airlight polarization model based on RT3 atmospheric radiative transfer model is also suitable for the haze weather.

3.2.2 Estimation of $A_{\infty}$ and $p$ : We obtain the polarization distribution of airlight through the airlight polarization model. Based on these data, we can easily estimate $A_{\infty}$ and $p$. We obtain the maximum intensity of the airlight as the estimation of $A_{\infty}$. And then, the degree of polarization of this airlight point is the estimation of $p$. If completely elimination of atmospheric scattering will lead to deep and dark sky region (Schechner et al., 2003). In order to correct the estimation error, a correction factor and a bias factor are introduced to revise $A_{\infty}$ and $p$

$$
\begin{gathered}
A_{\infty r}=A_{\infty} \times \varepsilon_{1} \\
p_{r}=p \times \varepsilon_{2}
\end{gathered}
$$

where $A_{\mathrm{s} o r}=$ corrected airlight radiance at infinite distance

$\varepsilon_{1}=$ correction factor

$p_{r}=$ corrected degree of polarization of the airlight

$\varepsilon_{2}=$ bias factor

The improvement of image dehazed quality and the control of noise by slightly enlarging the value of $A_{\infty}$ and $p$. The value of $\varepsilon_{1}$ must make sure that $A_{\triangleright \circ}<I$, and in order to ensure $p_{r}$ in a reasonable range $\left(0<p_{r}<1\right)$, the value of $\varepsilon_{2}$ should be $1<\varepsilon_{2}<1 / p$. After achieved the $I^{\perp}, I^{\|}, A_{\infty}$ and $p$, we can obtain the dehazed image by the Eq. (1).

\section{EXPERIMENTAL RESULTS AND DISSCUSSION}

The multi-spectral full-polarization imager used in experiment can work under 400-700nm, has 476nm, 514nm, 530nm, 647nm, $676 \mathrm{~nm}$ five different spectral bands. Four different polarization images of five spectral bands in horizontal detection mode can be obtained by the imager. The images were collected in horizontal detection mode on New Main Building of Beihang university in March 28 2018, where the geographical coordinates are $116^{\circ} 30^{\prime}$
E, 399 ${ }^{\prime}$ N. The Air Quality Index (AQI) was 248. Meanwhile, atmospheric aerosol optical thickness (AOT) was measured by a hand-held multi-band sun photometer (MISROTOPS II) shown in Table 1.

\begin{tabular}{|c|c|c|c|}
\hline \multicolumn{4}{|c|}{ AOT in different wavelength } \\
\hline $440 \mathrm{~nm}$ & $500 \mathrm{~nm}$ & $675 \mathrm{~nm}$ & $870 \mathrm{~nm}$ \\
2.787 & 2.747 & 2.714 & 2.500 \\
\hline
\end{tabular}

Table 1. AOT measured by MISROTOPS II

To demonstrate the effectiveness of the proposed method, results of Schechner was used for comparison. Schechner' method chose the sky area with the highest luminance value in the image to estimate $A_{\infty}$ and used the sky polarization difference information to compute $p$. The results images of Schechner' method and our proposed dehazing method are shown in Figure 4.

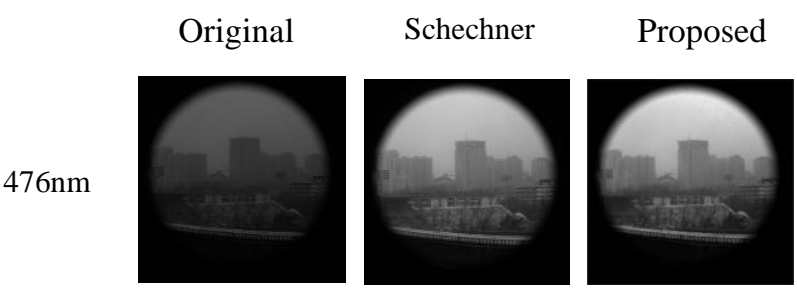

$514 \mathrm{~nm}$
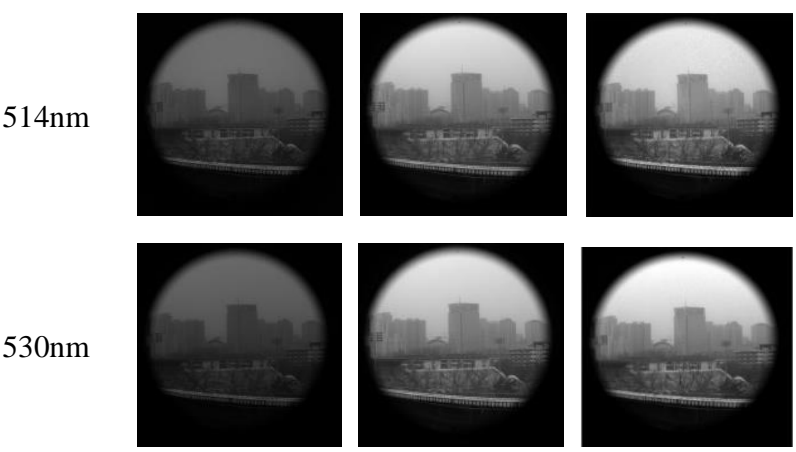

$647 \mathrm{~nm}$
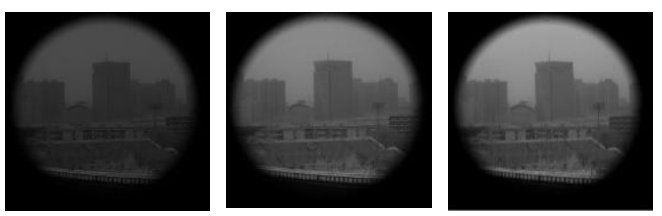

$676 \mathrm{~nm}$

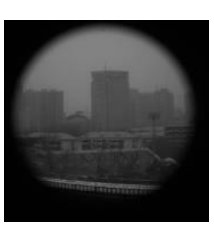

(a)

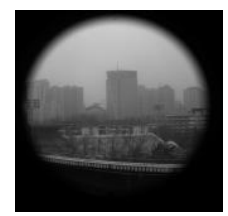

(b)

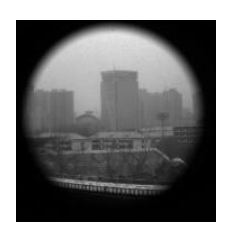

(c)
Figure 4. dehazed results using five spectral bands. (a) the first image of four different original polarimetric images, (b) Schechner's dehazed method, (c)the proposed dehazing method

The original polarization images under $476 \mathrm{~nm}, 514 \mathrm{~nm}, 530 \mathrm{~nm}$, $647 \mathrm{~nm}, 676 \mathrm{~nm}$ are shown in Fig. 4(a). The original images are attenuated by the heavy haze, the tall buildings in the far-field scene are almost submerged into the background and the clarity of the stadium in the near-field scene is affected. Also, we can observe that the far-field of the original images under $676 \mathrm{~nm}$ are clearer than others. From the results of the dehazed images from Schechner and proposed method, we observe that the clarity of 
the stadium is enhanced and it is easier to identify the wall detail of the tall buildings in the far-field scene.

Next, in order to evaluate our method in an objective manner, we use the image quality evaluation indexes to evaluate the reconstructed image quality, which are image information entropy $(\mathrm{H})$, gray standard deviation (e) and average gradient $(\mathrm{G})$ The scores of dehazing methods for different spectral bands images in Fig. 4 are listed in Table 2.

\begin{tabular}{|c|c|c|c|c|}
\hline $\begin{array}{c}\text { Spectral } \\
\text { band }\end{array}$ & Image & $\mathrm{H}$ & $\mathrm{e}$ & $\mathrm{G}$ \\
\hline \multirow{3}{*}{$476 \mathrm{~nm}$} & Original & 5.4357 & 31.7104 & 0.0031 \\
& Schechner & 6.1755 & 66.7777 & 0.0064 \\
& Proposed & 6.1819 & 79.4715 & 0.0087 \\
\hline \multirow{3}{*}{$514 \mathrm{~nm}$} & Original & 5.5923 & 40.3790 & 0.0044 \\
& Schechner & 6.3880 & 75.0675 & 0.0077 \\
& Proposed & 6.3129 & 82.9812 & 0.0104 \\
\hline \multirow{2}{*}{$530 \mathrm{~nm}$} & Original & 5.5677 & 35.4716 & 0.0036 \\
& Schechner & 6.4192 & 76.2932 & 0.0074 \\
& Proposed & 6.2989 & 85.1516 & 0.0090 \\
\hline \multirow{2}{*}{$647 \mathrm{~nm}$} & Original & 5.3022 & 26.7360 & 0.0025 \\
& Schechner & 5.9725 & 46.9980 & 0.0041 \\
& Proposed & 6.9945 & 81.4933 & 0.0194 \\
\hline \multirow{2}{*}{$676 \mathrm{~nm}$} & Original & 6.0371 & 44.7078 & 0.0043 \\
& Schechner & 6.6682 & 70.9603 & 0.0068 \\
& Proposed & 6.7412 & 71.3724 & 0.0083 \\
\hline
\end{tabular}

Table 2. Quantitative comparison of the 5 spectral bands for image quality evaluation indexes.

As can be observed from Table 2, the proposed method significantly improved image quality. Compared to the original image, the dehazed image has been greatly improved in image information entropy, gray standard deviation and average gradient. Compared to the Schechner's method, our dehazing method can outperform in terms of this metrics. We can conclude from these experimental results that our method has a better performance in raising image contrast and image clarity.

Our method is also useful in the polarization spectral images collected in vertical downward detection mode. The original images have less sky area. We have collected four polarization spectral images in vertical downward detection mode, and one of them is shown in Fig. 5(a). Using our proposed method, the dehazed image is shown in Fig. 5(b). From the comparison of these two images, it can be seen that the visibility of Fig. 5(b) is much better than Fig. 5(a).

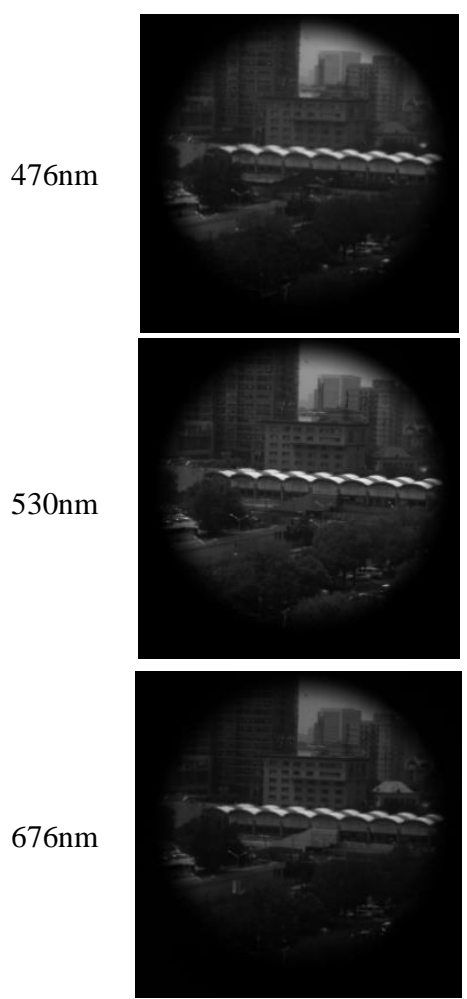

(a)
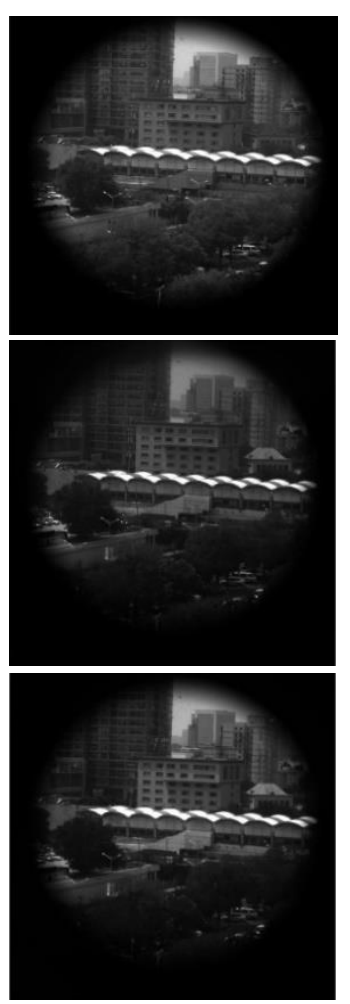

(b)
Fig. 5 dehazed results using three spectral bands. (a) the first image of four different original polarimetric images, (b) dehazed image

\section{CONCLUSIONS}

In this paper we proposed a new method to estimate $A_{\infty}$ and $p$. By using the airlight polarization model based on RT3 atmospheric radiative transfer model to simulate the polarization distribution of sky light, we estimate the airlight radiance at infinite distance and the degree of polarization of the airlight accurately, thereby obtaining a suitably dehazed image. It is important that the proposed method overcome the dependence of the sky area and the external interference of high-brightness objects and strong light sources when we estimate $A_{\infty}$ and $p$. The experiments are proved that our method affords dehazed images with rich detail not only in horizontal detection mode but also in vertical downward detection mode. It will provide a new way to extend operation distance of polarization imager systems and enhance its imaging detection performance in haze weather.

At present, we choose the point which has the maximum intensity of the airlight in the whole polarization distribution of airlight to estimate $A_{\infty}$ and $p$. In order to find a more suitable sky area to estimate these two parameters, we will divide up the polarization distribution of airlight obtained by the airlight polarization model in the future.

\section{ACKNOWLEDGEMENTS}

This work was supported by the National key R \& D project of China [Grant number 2017YFC0602104] and the National Natural Science Foundation of China [Grant number 61571029]. 


\section{REFERENCES}

He, K., Jian, S., Fellow, IEEE, Tang, X., 2011. Single Image Haze Removal Using Dark Channel Prior. IEEE Transactions on Pattern Analysis \& Machine Intelligence 33, 2341-2353.

Liang, J., Ren, L., Ju, H., Zhang, W., Qu, E., 2015. Polarimetric dehazing method for dense haze removal based on distribution analysis of angle of polarization. Optics Express 23, 2614626157.

Namer, E., Shwartz, S., Schechner, Y.Y., 2009. Skyless polarimetric calibration and visibility enhancement. Optics Express 17, 472-493.

Qi, M., Hao, Q., Guan, Q., Kong, J., Zhang, Y., 2015. Image dehazing based on structure preserving. Optik 126, 3400-3406.

Qu, Y., Zou, Z., 2017. Non-sky polarization-based dehazing algorithm for non-specular objects using polarization difference and global scene feature. Optics Express 25, 25004-25022.

Schechner, Y.Y., Averbuch, Y., 2007. Regularized Image Recovery in Scattering Media. IEEE Transactions on Pattern Analysis and Machine Intelligence 29, 1655-1660.

Schechner, Y.Y., Narasimhan, S.G., Nayar, S.K., 2001. Instant dehazing of images using polarization, Computer Vision and Pattern Recognition, pp. 325-332.

Schechner, Y.Y., Narasimhan, S.G., Nayar, S.K., 2003. Polarization-based vision through haze. Applied Optics 42, 511525.

Tan, R.T., 2008. Visibility in bad weather from a single image, Computer Vision and Pattern Recognition, pp. 1-8.

Tarel, J., Hautiere, N., 2009. Fast visibility restoration from a single color or gray level image, International Conference on Computer Vision, pp. 2201-2208.

Xia, P., Liu, X., 2016. Image dehazing technique based on polarimetric spectral analysis. Optik 127, 7350-7358.

Zhang, Y., Zhao, H., Cheng, X., Xiong, S.J., 2011. Design of Full-Polarized and Multi-Spectral Imaging System Based on LCVR. Spectroscopy and Spectral Analysis 31, 1375.

Zhu, Q., Mai, J., Shao, L., 2015. A Fast Single Image Haze Removal Algorithm Using Color Attenuation Prior. IEEE Transactions on Image Processing 24, 3522-3533.

Zhang, Y., Zhang, Y., Zhao, H., 2017. A Skylight Polarization Model of Various Weather Conditions. J. Infrared Millim. Waves 36(4), 217-223. 